\title{
A enfermeira gestora de casos na Espanha: enfrentando o desafio da cronicidade por meio de uma prática integral
}

\author{
Case management nurse in Spain: facing the challenge of chronicity \\ through a comprehensive practice
}

Helena Maria Scherlowski Leal David (https://orcid.org/0000-0001-8002-6830) ${ }^{1}$

José Ramón Martínez Riera (https://orcid.org/0000-0002-4926-6622) ${ }^{2}$

Antonia Herraiz Mallebrera (https://orcid.org/0000-0001-6942-7967) ${ }^{3}$

Maria Fernanda de Lima da Costa (https://orcid.org/0000-0002-1077-1381) ${ }^{4}$
${ }^{1}$ Departamento de Enfermagem de Saúde Pública, Faculdade de Enfermagem, Universidade do Estado do Rio de Janeiro. Boulevard 28 de Setembro $157 / 7^{\circ}$, Vila Isabel. 20551030 Rio de Janeiro RJ Brasil.helenalealdavid@ gmail.com

${ }^{2}$ Faculdade de Ciências da Saúde, Departamento de Enfermagem Comunitária, Medicina Preventiva e Saúde Pública e História das Ciências, Universidade de Alicante. Alicante Espanha.

${ }^{3}$ Sistema de Saúde do País Basco. Vitória-Gasteiz Pais Basco Espanha.

${ }^{4}$ Sistema de Saúde da Andaluzia, Hospital Geral de Málaga. Málaga Espanha.

\begin{abstract}
A new type of nurse role was established in the Spanish health care system in the late 1990s, currently called case manager nurse, to ensure access to resources needed to cope with chronic conditions and comprehensive home care. This paper aims to present this figure and discuss aspects of its work. The methodology was based on the bibliographic review of papers and normative publications and interviews with primary health care nurses, nursing faculty, and case management nurses. We present a brief history of the implementation of the case manager nurse role and the conceptual and operational bases of her practice in three autonomous Spanish communities: Andalusia, Valencian Community, and Basque Country, discussing potentialities and issues concerning this practice. In the conclusions, we make some considerations on the possible implementation of the case manager nurse in the Brazilian health system.
\end{abstract}

Key words Nurses, Chronic diseases, Health care system, Professional practices
Resumo Em fins da década de 1990, instituiuse um novo tipo de atuação para a enfermeira no sistema de saúde espanhol, denominada atualmente de enfermeira gestora de casos, com vistas à garantia do acesso aos recursos necessários para o enfrentamento das condições de cronicidade e atendimento integral no domicílio. $O$ objetivo do artigo é o de apresentar essa figura e discutir aspectos de sua atuação. A metodologia baseouse em revisão bibliográfica de artigos e publicações normativas e entrevistas com enfermeiras de Atenção Primária de Saúde, docentes de enfermagem e enfermeiras gestoras de casos. Apresenta-se um breve histórico de implantação da enfermeira gestora de casos e as bases conceituais e operacionais de sua prática em três comunidades autônomas espanholas: Andaluzia, Comunidade Valenciana e País Basco, discutindo potencialidades e questões a respeito dessa atuação dentro de um sistema de saúde de características universais. Nas conclusões, são tecidas algumas considerações a respeito das possibilidades para a implantação da figura da enfermeira gestora de casos no sistema brasileiro de saúde.

Palavras-chave Enfermagem, Enfermidades crônicas, Sistemas de saúde, Prática profissional 


\section{Introdução}

Em fins dos anos 1990, na Comunidade Autônoma espanhola de Canárias, foi desenvolvido um processo de reorganização do trabalho da enfermagem na Atenção Primária de Saúde (APS), com a definição de novos protocolos, gestão de recursos materiais e humanos e comunicação entre os níveis da APS e Atenção Hospitalar (AH). Os elementos desencadeadores estavam em curso, dado o perfil de envelhecimento da população e aumento das condições de cronicidade, além da alta dispersão territorial nas Canárias. Algumas enfermeiras da APS assumiram esse papel mediador, como "resposta à necessidade de potencializar os serviços de Atenção Domiciliar", passando a atuar como gestoras de cuidados. Foi primeiramente denominada enfermera de enlace, cuja tradução seria enfermeira de ligação, ou de mediação ${ }^{1}$.

No decorrer dos anos seguintes, além de se expandir para o sistema de saúde espanhol, esta figura passou a se denominar enfermeira gestora de casos (EGC). Aqui cabe explicar a opção de uso do termo enfermeira, com flexão de gênero no feminino: é a forma adotada nos países de língua espanhola, e que reflete a histórica feminização da profissão, pelo que optamos por mantê-la no texto em língua portuguesa.

As Canárias constituem-se em uma das 17 Comunidades Autônomas espanholas, consolidadas após a promulgação da Constituição Espanhola de 1978. Geopolítica, história e cultura, dentre outros aspectos, definem essas comunidades. Podem ser comparadas a estados ou unidade federativas, como no Brasil e outros países republicanos, embora com consideráveis diferenças no que tange à sua relação com o executivo federal. Os sistemas de saúde dessas comunidades, apesar das linhas gerais ditadas pela Lei Geral de Saúde espanhola de 1986, possuem um alto grau de independência de ação e proposição, o que também pode resultar em diferentes modelos organizativos do trabalho para os profissionais de saúde ${ }^{2}$.

A partir da experiência canária, outras Comunidades rapidamente adotaram o modelo da EGC. A Catalunha foi a comunidade autônoma que em seguida passou a incluir a enfermeira de enlace em seus quadros, seguida pela Andaluzia e Comunidade Valenciana e outras, sendo algumas de implantação mais recente, como o País Basco ${ }^{3}$.

A EGC não é um tipo de especialidade no âmbito da formação profissional, nem consta como cargo específico nos organogramas. Em al- guns locais existe a menção à EGC como prática ou competência avançada de enfermagem ${ }^{3}$, mas não pode ser considerada como uma especialista nesta modalidade de atuação e formação. Não parece haver na Espanha, até o momento, um entendimento consensual sobre o termo "prática avançada" que nos permita adentrar nesse debate.

Mesmo sem um padrão nacional de formação ou de inserção para a EGC, ela pode atuar na Atenção Hospitalar e/ou na APS, dentro de uma mesma comunidade autônoma.

Avaliações sobre o papel da EGC indicam que é um tipo de atuação com resolutividade e impactos positivos nos sistemas de saúde e para as pessoas portadoras de condições crônicas complexas. Configura-se como uma prática de características matriciais, com atuação transversal em relação aos níveis de atenção, mobilizando os diversos recursos de saúde de um dado território ${ }^{1,3}$.

O objetivo do artigo é apresentar e discutir aspectos centrais do processo de trabalho da EGC de três comunidades autônomas espanholas - Andaluzia, Comunidade Valenciana e País Basco, sintetizando os principais aspectos históricos, das atribuições profissionais e quanto aos resultados desse trabalho.

Após a descrição da metodologia, o texto estrutura-se em dois eixos: o primeiro trata brevemente da constituição da enfermagem espanhola como profissão, apresenta os principais elementos conceituais da proposta da EGC, e descreve sua trajetória nas comunidades selecionadas.

No segundo eixo, articulamos alguns resultados de avaliações e pesquisa a relatos obtidos junto a enfermeiros, gestores e pesquisadores entrevistados.

Nas conclusões, tecemos algumas reflexões sobre o que podemos aprender dessa experiência, arriscando breves considerações sobre a eventual inserção da EGC na realidade brasileira.

\section{Metodologia}

O estudo é um recorte do projeto de pesquisa "A enfermagem de Atenção Primária de Saúde em uma perspectiva comparada entre Brasil e Espanha: Conjunturas de crise e impactos na saúde". Inclui duas abordagens metodológicas: revisão bibliográfica e análise qualitativa de entrevistas.

Foi realizada, primeiramente, uma pesquisa de revisão bibliográfica incluindo artigos, relatórios técnicos e documentos normativos de três Comunidades Autônomas - Andaluzia, Comu- 
nidade Valenciana e País Basco. Pesquisou-se nas bases de dados Scielo, Cuiden e CINAHL, por meio de busca simples, e nas páginas oficiais dos sistemas sanitários. Os descritores utilizados foram, em espanhol, "enfermera gestora de casos", "gestora de casos em España", "enfermeira de enlace", tendo sido considerados como critérios de seleção de textos que : i) descrevessem experiências com enfermeras gestoras de caso ou enfermeras de enlace; ii) apresentassem resultados de avaliações da atuação da EGC e iii) descrevessem as atribuições da EGC . Foram selecionados 10 artigos científicos e 06 textos técnicos oficiais e/ ou manuais, com descrição de atribuições, formação, e trazendo indicadores de avaliação sobre a atuação da enfermeira gestora de casos dos sistemas de saúde das Comunidades de Andaluzia, Valenciana e País Basco.

A abordagem qualitativa baseou-se na produção e análise de entrevistas individuais realizadas com 7 enfermeiras das áreas da Atenção Primária (2), investigadoras e docentes de enfermagem (2), enfermeiras gestoras de caso (2), e enfermeira coordenadora de APS (1). Os participantes foram selecionados pelo método de snowballing, após contato inicial com enfermeira docente, e com enfermeiras que já haviam sido entrevistas em outras etapas do projeto. Os critérios de inclusão foram os de conhecer o trabalho da EGC e aceitar participar do estudo, e a amostra foi definida por saturação, em função da recorrência de informações.

As entrevistas foram realizadas presencialmente, em centros de saúde, hospitais e universidades. Houve uma entrevista por e-mail, nesse caso solicitando o envio de arquivo de áudio de resposta às perguntas. Todos os participantes assinaram o Termo de Consentimento Informado, após explicações pelos pesquisadores sobre o estudo, gravadas e posteriormente transcritas. Todo o material transcrito foi enviado aos entrevistados para validação do conteúdo. $\mathrm{O}$ estudo foi submetido e aprovado pelos Comitês de Ética em Pesquisa com Seres Humanos da Universidade do Estado do Rio de Janeiro e da Universidade de Alicante, Espanha.

Tanto a coleta de dados bibliográficos como as entrevistas tiveram lugar durante os meses de janeiro e fevereiro de 2019, em território espanhol, nas Comunidades de Andaluzia, Valenciana e País Basco.

\section{Enfermeira gestora de casos- buscando garantir a continuidade de cuidados}

É notável o desenvolvimento social e econômico da Espanha, após os difíceis anos do franquismo. A expectativa de vida, de 82,83 anos em 2018, segundo dados do Banco Mundial, é uma das mais altas da Europa, e supera a média mundial, de 72,04 anos. Mudanças sociodemográficas, culturais e nos modos de viver se expressam em alta prevalência de problemas crônicos de saúde, com impacto operacional e financeiro no sistema de saúde, representando o maior volume de demandas de saúde ${ }^{4}$.

Após a Reforma Sanitária de 1986, o sistema de saúde estruturou-se em dois níveis: a Atenção Primária de Saúde, de acesso universal, e a Atenção Hospitalar ou Especializada ${ }^{5}$.

A enfermagem, que era fragmentada em diversas profissões, teve seu perfil consolidado no ano de 1976 . Foi um período marcado por mobilizações conhecidas como la revolución de las batas blancas, exigindo a formação de nível superior dentro das universidades ${ }^{6}$. As enfermeiras, já fortalecidas e organizadas, participaram ativamente no posterior processo de reforma sanitária, que deu origem à Lei Geral de Saúde de 1986.

É o tema da continuidade de cuidados que leva às primeiras proposições em torno da figura da enfermera de enlace em Canárias. Garantir a continuidade dos cuidados às pessoas que estiveram internadas ou necessitam de atenção especializada no domicílio é problemático num território ultramarino e de grande dispersão geográfica e populacional. Na implantação do Programa de Atenção Domiciliar (desenvolvido pela APS), discussões sobre como ampliar a atuação e a resolutividade do nível primário resultaram nas primeiras experiências com a enfermeira de enlace. Vale ressaltar que Enfermagem Familiar e Comunitária é uma área de atuação essencial na enfermagem espanhola, e se caracteriza pelo enfoque do cuidado às necessidades de saúde de indivíduos, famílias e comunidades (e não apenas atender a pessoas enfermas), por meio de uma prática integrada (ao sistema de saúde), integradora (de recursos e pessoas) e integral (em relação aos níveis de atenção e às necessidades de saúde $)^{7}$.

Coordenar os cuidados, no primeiro programa desenvolvido em Canárias, incluía: cadastrar e avaliar todas as pessoas com mais de 65 anos de idade; favorecer a atenção domiciliar a toda pessoa incapacitada ou de risco; manter e melhorar a qualidade de vida do cuidador domiciliar princi- 
pal da pessoa incapacitada; facilitar a prestação de cuidados domiciliares para a equipe de Atenção Primária; criar e/ou manter mecanismos de relação entre a equipe de Atenção Primária e a rede de serviço social; conhecer e coordenar mecanismos de relação com os demais níveis assistenciais; potencializar e facilitar a coleta e reutilização de equipamentos usados na atenção domiciliar e avaliar os impactos econômicos da proposta ${ }^{8}$.

$O$ conceito de continuidade de cuidados não é consensual. Alguns autores se referem a uma dupla dimensão da ideia de continuidade assistencial: uma, de características verticais, diz respeito à relação entre níveis de atenção, como a continuidade de cuidados entre o hospital e o domicílio; outra, de caráter pessoal-longitudinal e através do tempo parte de uma concepção ampliada sobre o cuidado, incluindo as ações de promoção da saúde e prevenção9 .

A continuidade de cuidados pode ser entendida como:

[...] un concepto central que favorece el bienestar y el funcionamiento de los pacientes en función del grado de atención que necesitan, facilita la efectividad de los diferentes servicios, está coordinada eficazmente entre diferentes profesionales y organizaciones y con relación al tempo y permite dar una respuesta a las necesidades de las personas con problemas de salud ${ }^{8}$.

As três Comunidades Autônomas estudadas adotaram elementos conceituais e operacionais comuns para a figura da EGC. Além da continuidade de cuidados no tempo e entre níveis de atenção, outros temas foram considerados, tais como a atenção a grupos populacionais vulneráveis; a mediação entre as necessidades das pessoas, famílias e cuidadores e a capacidade de resposta do sistema de saúde; a racionalização e otimização de recursos, o planejamento e a antecipação de problemas, complicações ou consequências; a ampliação do cuidado digno e de qualidade por meio de assistência do tipo hospitalar prestada no domicílio; produção e manutenção do fluxo de informações sobre pessoas, cuidadores e famí$\operatorname{lias}^{10}$.

A Andaluzia desenvolveu de forma rápida e ampliada um modelo de gestão de casos, logo na primeira metade da década de 2000, que posteriormente colaborou para nortear outras comunidades autônomas. Houve apoio institucional e a formulação de um plano concreto de atenção para pessoas incapacitadas e idosas, elementos iniciais que ajudaram a impulsionar a proposta ${ }^{1}$.

O termo gestão de casos é adotado a partir das experiências de países como o Reino Unido,
Estados Unidos e Canadá (case management), e vem sendo desenvolvido desde a década de 60, inicialmente na área de saúde mental ${ }^{1,9}$, e posteriormente em outras áreas, porém sem designar uma categoria profissional específica para assumir a função gestora. Ainda assim, vale apontar que, na maioria dos países que implantou processos de gestão de casos, esta função tem sido desenvolvida por enfermeiras.

O ano de 2002 é o marco da implantação no sistema andaluz da enfermeira de enlace comunitária, e em 2003, a hospitalar. Já em 2006, o governo andaluz publica o Manual da Enfermeira Gestora de Casos no Hospital ${ }^{11}$, e em 2007, o Manual da Enfermeira Gestora de Casos de Atenção Primária ${ }^{12}$, demarcando a atuação concomitante dessas duas figuras.

Qual a distinção entre as enfermeiras gestoras da APS e hospitalar na experiência andaluza? A EGC da Atenção Primária, também denominada de Enfermeira Gestora de Casos Comunitária, tem como público-alvo pessoas vinculadas a um centro de saúde, e que necessitam de cuidados domiciliares. Os objetivos da sua atuação incluem manter e melhorar a qualidade de vida de toda pessoa incapacitada ou em risco de sofrer incapacidade, assim como a de seus cuidadores; facilitar a melhora da atenção domiciliar prestada pela equipe de APS, a coordenação desta equipe com a rede de apoio social, e melhorar a coordenação com outros níveis, para a garantia da continuidade de cuidados ${ }^{13}$.

Já a EGC Hospitalar tem como população -alvo pessoas internadas no hospital, e que, em função da complexidade do seu estado de saúde, requerem a coordenação de recursos referentes a profissionais/especialidades diversas para a continuidade de seus cuidados no domicílio, atendendo também às necessidades dos seus cuidadores. Os problemas mais comuns são os osteomusculares e de fraturas, cardiovasculares incapacitantes, transtornos mentais graves, HIV -AIDS e Doença Pulmonar Obstrutiva Grave.

A EGC da APS capta seus pacientes e cuidadores a partir das informações dos serviços de APS e suas equipes, enquanto a EGC Hospitalar realiza busca ativa nos hospitais, além de receber comunicações de outros enfermeiros e profissionais. A gestão de recursos hospitalares a serem usados no domicílio também é responsabilidade da EGC Hospitalar.

A experiência da Comunidade Valenciana se deu pouco após a andaluza, na primeira metade da década de 2000. Passa a integrar o Plano de Melhora da Atenção Domiciliar entre 2004 e 
2007, da Consellería de Sanitat (Sistema de Saúde Valenciano), primeiramente como Enfermera de Gestión Domiciliaria (Enfermeira de Gestão Domiciliar). Como ECG, é implantada de modo experimental nos Departamentos valencianos de Castellón e de Alicante, neste último dentro do Hospital Geral, como Enfermera de Enlace Hospitalaria $^{14}$. O resultado positivo desse projeto piloto levou o Sistema Valenciano de Saúde a incorporar a figura da Enfermeira Gestora de Casos como parte da Estratégia de Atenção a Pacientes Crônicos e do Modelo de Atenção Integrada de Casos Complexos. Também está incluído o cuidador familiar como público-alvo, sendo os atributos que definem a necessidade de uma intervenção por parte da EGC: a complexidade clínica; a complexidade de manejo no domicílio e na comunidade; elevado risco de urgências e internação hospitalar ${ }^{15}$.

A proposta valenciana é a de inserir a EGC dentro de um modelo de atenção integrada, para que o cuidado possa ser exercido em qualquer espaço. Há um apoio institucional forte, evidenciado por uma instância-chave nesse processo, a Unidade de Hospital a Domicílio (UHD), que se constitui em um programa de atenção domiciliar de base hospitalar, em permanente diálogo e comunicação com a Atenção Primária. Assim como na Andaluzia, há a Enfermeira Gestora de Casos Comunitária, na APS, atuando nos domicílios, e a Enfermeira Gestora de Casos Hospitalares, vinculada ao Hospital, que engloba a atenção hospitalar a casos agudos (urgências/emergências) e casos crônicos, e o chamado hospital a domicílio. Ambas as figuras dão suporte à gestão de casos crônicos complexos no nível departamental, podendo abarcar, cada uma vários Centros de Saúde e serviços hospitalares, a depender da dispersão geográfica. Em termos numéricos, a evolução da sua inserção no Sistema de Saúde Valenciano era de 9 EGC Comunitárias e 4 Hospitalares, em 2010, em dois departamentos, chegando, em 2017, a 53 EGC Comunitárias e 22 Hospitalares. Um aspecto que está bastante destacado nos relatórios técnicos é o apoio ao cuidador familiar principal, por meio de cursos específicos e a manutenção de uma relação estreita entre a EGC e estas pessoas.

A terceira experiência, que é também a mais recente, é a da implantação da figura da EGC no País Basco, que se deu a partir de 2014. Esta comunidade autônoma, de características sociais, culturais e políticas bastante diferenciadas em relação ao restante do país vem implantando programas com vistas ao enfrentamento das condições de cronicidade, que resultam em comorbidades e casos complexos. No documento Estrategia para afrontar el reto de la cronicidad en Euskadi (Estratégia para enfrentar a cronicidade no País Basco), de 2010, além do levantamento acerca do impacto epidemiológico e social dos problemas crônicos há um forte estímulo à participação comunitária no processo, além do reconhecimento do protagonismo da pessoa portadora de problemas crônicos, expresso por meio dos conceitos de paciente ativo, de envelhecimento ativo e estímulo à autonomia ${ }^{16}$.

Uma primeira modelagem realizada em 2008 para a inclusão da EGC previu três figuras, duas em APS (uma para pacientes crônicos, a Enfermeira Gestora de Continuidade, e outra para pacientes crônicos complexos), e a EGC Hospitalar. Após avaliação, permaneceram duas figuras - a EGC de Ligação (Enlace) Hospitalar e a Enfermeira Gestora de Competência Avançada ${ }^{17}$. Entre 2010 e 2012, a ideia era a de formar 300 enfermeiras em competências avançadas para a gestão de casos, em especial de casos complexos ${ }^{18}$.

O que se denomina de competências avançadas é descrito como um processo de qualificação profissional para o desenvolvimento de novas competências, por meio de uma metodologia participativa, envolvendo atores institucionais, da academia e outros ${ }^{16,18}$.

Há um foco mais evidente no enfrentamento do adoecimento e dos casos complexos, sendo a EGC definida como "a profissional que realiza a gestão de casos para dar respostas às necessidades de atuação de forma proativa e coordenada para identificar as pessoas mais vulneráveis, em situações e com patologias complexas, o que implica em um esforço coordenado e em estreita colaboração com o interessado e seus cuidadores”. Está também explicitado que não se trata de criar novas categorias profissionais, e sim a de estabelecer uma diferenciação na funcionalidade dos diferentes postos de trabalho, para o desenvolvimento das respectivas competências ${ }^{18}$. Dentre as três experiências, é a do País Basco a que apresenta, nos documentos, uma reflexão mais consolidada a respeito da figura da EGC.

A enfermeira espanhola tem garantida a formação de nível superior há relativamente pouco tempo quando se compara com o Reino Unido ou com o Brasil. É digno de nota a categoria ter obtido visibilidade e reconhecimento suficientes para propor a inserção de uma nova figura, de características mediadoras e, sem dúvida, de alta capacidade decisória quanto aos projetos terapêuticos para os portadores de problemas 
crônicos. No entanto, existem tensões entre os campos das competências das diversas categorias profissionais.

\section{Avaliações e relatos sobre a Enfermeira Gestora de Casos}

Um informe de imprensa do ano de 2016, emitido pelo Conselho Geral de Enfermagem da Espanha, traz como título uma assertiva: La gestión de casos em enfermeira: uma grán desconoci$d a^{19}$. Levando-se em conta os quase vinte anos de implantação da EGC no país, é de estranhar que essa função ainda seja considerada desconhecida entre as enfermeiras, o que leva a indagações a respeito de como tem sido incorporada essa função e qual o seu grau de institucionalidade.

Logo após as primeiras experiências, parece ter havido (e ainda há) consenso suficiente em torno da positividade da atuação da EGC, independente dos tipos e nomes adotados - comunitárias, de APS, hospitalares, de competências avançadas ou qualquer outro. A carga de trabalho exigida para enfrentar a cronicidade parece ser desproporcional à velocidade e gravidade no avanço destas condições, o que torna bem-vinda qualquer proposta que melhore a coordenação dos processos e recursos. Ao mesmo tempo, a cobertura desses serviços é instável, e a formação varia entre as comunidades.

Do ponto de vista epidemiológico, estudos diversos foram desenvolvidos com vistas a avaliar os impactos do trabalho da EGC, mas poucos comparam o antes e o depois. Na Andaluzia, há um predomínio de estudos descritivos, com foco em casos específicos, que apresentam a prática da EGC em situações crônicas como problemas hematológicos ${ }^{17}$, diabete ${ }^{20}$, e HIV-AIDS ${ }^{21}$. Todos trazem uma avaliação qualitativa geral em termos de satisfação, diminuição da carga de trabalho de cuidadores, otimização de tempo e recursos. Estudos mais sofisticados foram desenvolvidos, alguns com desenho quase-experimental, e com resultados estatisticamente significativos quanto à diminuição da sobrecarga mensurável de cuidadores. Foram mensurados resultados favoráveis no grau de satisfação dos pacientes e quanto 'a racionalização na utilização dos recursos no grupo que sofreu a intervenção da EGC quando comparado ao grupo-controle, sem intervenção ${ }^{22}$.

Os relatórios e programas técnicos produzidos pelos sistemas de saúde das comunidades estudadas trazem dados quanto aos impactos positivos da intervenção. Na Andaluzia apresenta-se uma avaliação positiva: melhoras na capacidade funcional, melhor manejo de medicação e redução na institucionalização e uso de serviços pela população idosa; redução de reinternações hospitalares e do número de dias de internação de pessoas com demências; melhoras na aderência ao tratamento e na integração social, no bem-estar psicológico e qualidade de vida de pessoas com câncer; diminuição da hospitalização de pessoas com transtornos mentais graves, dentre outros $^{16}$.

$\mathrm{Na}$ Comunidade Valenciana, destacaram-se indicadores relativos às internações de urgência entre pessoas portadoras de problemas crônicos sob o controle da EGC hospitalar, com diminuição de 77\% em relação aos 12 meses anteriores ao projeto piloto de 2007-2010, assim como a diminuição de $70 \%$ no número de internações nesse grupo. Dentre os impactos positivos que foram atribuídos ao trabalho da EGC Comunitária estão o aumento na identificação de pacientes domiciliares "ocultos", aqueles que precisavam de cuidados domiciliares porém ainda não haviam sido identificados pelas equipes de APS, e a realização de cursos para pacientes e para cuidadores familiares ${ }^{15}$. No País Basco, as avaliações, ainda que mais recentes, também demonstram a diminuição de internações hospitalares nos grupos cobertos, em algumas comarcas, e indicadores de satisfação positivos entre pacientes, familiares e cuidadores $^{18}$.

No entanto, é preciso haver cautela na leitura dos indicadores, já que a avaliação deste tipo de intervenção não é simples, e tampouco há indicadores consolidados para tal. Uma questão é a que diz respeito à "exclusividade" de atuação de enfermeiras como gestoras de casos em relação às demais profissões. Atribuir o sucesso (e também o eventual fracasso) desta iniciativa apenas a uma categoria profissional é um simplismo, já que, ao planejar, mobilizar e gerir recursos e processos, se permite que outros profissionais ampliem sua atuação nos domicílios, somando forças e competências em prol da melhoria e da continuidade de cuidados ${ }^{22}$.

Por outro lado, não é ao acaso que na Espanha e em outros países que adotam modelos de gestão de casos são as enfermeiras os profissionais mais mobilizados para esta intervenção. A enfermeira, cuja formação básica é orientada por princípios ético-políticos e técnicos em favor de uma atuação integradora e educativa, acaba por desenvolver competências profissionais que a tornam elegível para atuações em frentes de trabalho que exigem capacidade de mediar conflitos e estabelecer processos dialógicos, sendo também 
uma categoria com forte aderência à cultura institucional nos sistemas de saúde, de modo geral ${ }^{23}$.

Os relatos das entrevistas trazem elementos importantes a serem considerados, quando analisados em conjunto com a bibliografia acadêmica e normativa.

Do ponto de vista do processo de implantação, é interessante observar que os relatos, embora não desconsiderem as influências das experiências de outros países, trazem como motivação principal o enfrentamento de condições concretas, que estes profissionais bem conheciam no seu dia a dia:

Por un lado, tenemos las enfermeras que buscan dar unos cuidados de excelencia y por otro tenemos un sistema que lo que intenta es que el paciente esté el menor tiempo posible en el hospital, que se den altas lo más precozmente posible, por lo que supone de beneficios para el paciente y de beneficios económicos para el sistema. Porque cualquier hospitalización que se alargue va a suponer un aumento de gastos, un aumento de complicaciones $y$ un aumento en fin de dinero, no? (Enfermeira de APS, País Basco).

Nosotros estudiamos mucho, leímos mucho, investigamos, y lo diseñamos. Pero no había como irnos a otros países a mirlarlos. Y tampoco había en aquel momento un modelo muy claro (Docente e pesquisador, Canárias).

Entonces la enfermera gestora de casos adquirió otro rol, no solo era la comunicación entre primaria $y$ hospitalizada, sino que ella llevaba esos pacientes complejos paliativos que necesitaban una serie de recursos, y poner eso en comunicación a todos los recursos para darle la prestación que el paciente necesitaba. Se dedicó a esos pacientes que requieren una movilidad de recursos especiales (Enfermeira de APS, Comunidade Valenciana)

Para a enfermeira que atua na coordenação da APS, há o desejo de que se possa ter o maior número possível de EGC:

Es una figura fundamental, y que debería haber por lo menos una en cada centro de salud. Esta enfermera gestora es la que va por delante. Por delante, $y$ va al domicilio, o sin domicilio, que pueden haber pacientes complejos, Y es la primera que hace una valoración in situ del problema, vale, hace una valoración tanto clínica como comunitaria de uno que está viviendo en una área. (Enfermeira coordenadora de APS da Comunidade Valenciana).

Esta enfermeira atua em uma província do interior, pequena, e que vem sofrendo um esvaziamento populacional, o que torna a população de idosos proporcionalmente muito maior que a de jovens, e, em decorrência, são os problemas crônicos os que mais impactam no sistema de saúde, sendo que os recursos sociais tendem a diminuir com o esvaziamento populacional.

A proposta da EGC também respondeu a necessidades percebidas de melhorar os fluxos e registro de informações sobre as pessoas e cuidadores familiares:

$Y$ además con los cuidadores familiares era un tema fundamental, pues no había registro, no había censo, no se sabía, no estaban valorados, no sabemos en qué situación estaban de salud esos cuidadores familiares, qué necesidades tenían, nada. Entonces, eso, por una parte, y luego por parte de los pacientes, pues también había mucho déficit de registro. (EGC, Comunidade Valenciana).

O processo de implantação da EGC não tem sido livre de conflitos e dificuldades. O primeiro grande conflito se dá no âmbito das relações interprofissionais e interpessoais: uma figura nova, com uma função diferenciada, inserida normativamente, a partir da gestão:

Cuál es el dilema que se genera? El dilema es el siguiente: como se suele hacer en este país, aqui que se dice poner el carro delante de los bueyes. Es decir, se generan figuras, se implantan, no se la explica adecuadamente, genera conflictos en el interior de los equipos. Con las enfermeras comunitarias, que dicen: y para qué viene esta enfermera si esto yo ya lo estoy haciendo? Y además me va a decir lo que tengo que hacer. No se explica adecuadamente y por lo tanto se generan problemas. Pero sí que es cierto que si está bien desarrollado suele dar buenos resultados. Y que cuando se conoce, se minimizan los riesgos de conflictos. (docente e pesquisador universitário, Comunidade Valenciana).

Este relato chama a atenção para o desafio de identificar um determinado perfil pessoal, de maturidade emocional e capacidade de mediação que confere a uns, mas não a outros, a capacidade de implementar processos de reorientação de práticas. O sucesso ou o fracasso de uma experiência como a da EGC pode ser também, em parte, atribuído a uma maior ou menor capacidade de negociação e de estabelecer relações de confiança. Isto aponta para um perfil profissional no qual competências clínicas e gerenciais precisam estar, necessariamente, articuladas a competências relacionais e de mediação de conflitos.

Alerta-se, ainda, que é imprescindível que os recursos necessários para a gestão de casos já existam ou estejam de alguma forma disponíveis:

En vez de crear un servicio para esos pacientes, que es la tendencia natural de los sistemas sanitarios, lo que se hace es poner una enfermera que coordine los servicios que están al de redor. [...] 
En muchos casos lo que se llama gestión de casos efectivamente no lo es. (Docente e pesquisador, Canárias).

Não se trata, portanto, de apenas alocar um profissional para apenas avaliar clinicamente os chamados casos crônicos complexos. O papel da EGC é o de, sobretudo, conseguir que serviços e profissionais atuem de forma coordenada e integrada.

$\mathrm{Na}$ ausência ou deficiência desses serviços, ou ainda nas equipes em que, por alguma razão, os profissionais não estão articulados, a EGC pode ter de assumir tarefas assistenciais, o que escapa às suas atribuições;

Si tú por el contrario te encuentras con equipos de enfermería dónde hay carencia, pues, porque hay a lo mejor falta de motivación, falta de responsabilidad, pues, entonces el trabajo es más intenso, de decir, es más intenso es que a veces tenemos que asumir parte del trabajo de cuidados más que de gestión del caso [...] (EGC, Andaluzia).

Os entrevistados afirmaram que o entendimento e reconhecimento da proposta dependem, também, de uma capacitação adequada das enfermeiras, necessidade também apontada nos documentos, embora não haja, até o momento, um modelo consensual de curso ou capacitação para EGC.

\section{O que podemos aprender da experiência espanhola de gestão de casos?}

Partimos de uma perspectiva ético-política que defende que a enfermagem é mais que trabalho ou profissão, e que se constitui em uma prática social imprescindível para a garantia do acesso universal e o enfrentamento das iniquidades em saúde. É preciso interrogar sobre o que podemos, como enfermagem brasileira, aprender da experiência originada na enfermagem espanhola, tendo em vista que compartilhamos, e iremos compartilhar de modo crescente, dos desafios da atenção às condições de cronicidade.

Um primeiro elemento a se destacar é o entendimento do processo de saúde-enfermidade como resultante da interação complexa de fatores, em diversos níveis da vida. O processo de implantação da figura da EGC na Espanha se ofe- rece como panorama emblemático para pensar a importância da prática da enfermeira para além do cuidado individual, já que o foco da atuação é amplo e inclui o paciente com enfermidade crônica, seus cuidadores principais, profissionais e serviços. É um tipo de atuação que corre o risco, no entanto, de ser capturada pela pesada burocracia dos processos necessários para mobilizar recursos.

Parece haver um "perfil desejado" para a EGC que envolve o desenvolvimento de competências de liderança e capacidade de articulação e mediação. Além disso, é preciso que outros profissionais da equipe e dos serviços conheçam a proposta, à qual espera-se uma aderência suficiente para que a capacidade e mobilizar recursos se efetive. Não se trata de uma condição dada, e sim a ser construída em cada nível local, a partir de um consenso mínimo entre gestão, serviços e profissionais de saúde.

A emergência de uma função profissional, que tem sido denominada "figura", como a da EGC aponta para uma opção exitosa, porém não totalmente isenta de um certo custo político e operacional. Supondo-se, como exercício imaginativo, que se tente implementar uma proposta como essa no Brasil, num sistema ou subsistema local, com recursos e treinamento minimamente adequados, ainda assim é preciso perguntar: quais as possibilidades de que o trabalho de uma EGC se desenvolva com a autonomia e velocidade necessárias diante das barreiras administrativas, burocráticas e corporativistas?

A questão da existência de um conjunto mínimo de serviços a serem geridos é outro fator a se considerar, dado que no modelo brasileiro os arranjos organizativos locais podem ser muito diferentes, assim como seus recursos. Por outro lado, a figura da EGC aponta para a possibilidade de estruturação de novos processos, e para a descoberta de recursos comunitários e institucionais desconhecidos ou sub-utilizados.

A experiência espanhola, portanto, pode servir colaborar para a permanente necessidade de criar e recriar um sistema sanitário capaz de dar respostas resolutivas e de qualidade para as necessidades em saúde. 


\section{Colaboradores}

HMSL David foi responsável pela concepção e desenvolvimento do estudo e do artigo. JRM Riera, AH Mallebrera e MFL Costa participaram apoiando a realização de entrevistas, a busca de material bibliográfico e a análise dos resultados.

\section{Agradecimentos}

O presente trabalho foi realizado com apoio da Coordenação de Apoio a Pessoal de Nível Superior - CAPES, fonte de financiamento 001.

\section{Referências}

1. Batres JP, Álvarez M, Gallardo P. De la precisión de cuidados a los cuidados imprescindibles. Las enfermeras gestoras de casos en Andalucía: la enfermera comunitaria de enlace. Rev Adm Sanit 2009; (7):313-322.

2. Bacigalupe A, Escolar-Pujolar A. The impact of economic crises on social inequalities in health: what do we know so far? Int J Equity Health [Internet]. 2014 [acessado 2018 Nov 18]; 25:13-52. Disponível em: https:// www.ncbi.nlm.nih.gov/pmc/articles/PMC4134120/. DOI: 10.1186/1475-9276-13-52

3. Sánchez-Martín CI. Cronicidad y complejidad: nuevos roles en Enfermería. Enferm Clin [Internet] 2014 [acessado 2019 Fev 20]; 24(1):79-89 Disponível em: http://www.sciencedirect.com/science/article/pii/ S1130862113001964)

4. Trescastro-López EM, Galiana-Sánchez ME, Bernabeu-Mestre J. Transición epidemiológica-nutricional y cambios demográficos en la España contemporánea. Panorama Social 2016; 23:167-175.

5. Abásalo I, Oinilla J, Negrín M.Equidad en la utilización de servicios sanitarios públicos por Comunidades Autónomas en España: un análisis multinivel. Revista de Economía Pública [Internet] 2008 [acessado 2019 Abr 2]; 187(4):87-106. Disponível em: https://sudocument. ulpgc.es/bitstream/10553/22108/1/Equidad_utilizaci\%C3\%B3n_servicios.pdf

6. Germán Bes C. A revolución de las batas blancas: la enfermería española de 1976 a 1978. Zaragoza: Prensa de la Universidad de Zaragoza; 2015.

7. Martínez-Riera JR. Enfermera Comunitaria. En: Martínez-Riera JR, Casado RP, organizadores. Manual Práctico de Enfermería Comunitaria. Barcelona: Elsevier; 2014. p. 214-221.

8. Llobregat MDS, Martínez-Riera JR. Continuidad de cuidados: nuevas figuras de gestión. En: Martínez-Riera JR, Casado RP, organizadores. Manual Práctico de Enfermería Comunitaria. Barcelona: Elsevier; 2014. p. 126-129.

9. Morales JM. Gestión de casos y cronicidad compleja: conceptos, modelos, evidencias e incertidumbres. Enferm Clin 2014; 24(1):23-34.

10. Leyva-Moral JM. Gestión de Casos: Aproximación Teórica. Rev ROL Enf 2008; 31(4):259-264.

11. España. Servicio Andaluz de Salud. Manual de la gestión de casos en Andalucía: enfermeras gestoras de casos en el hospital. Consejería de Salud. [Internet] 2006 [acessado 2019 Mar 1]. Disponível em: http://www.index-f. com/lascasas/documentos/lc0246.pdf

12. España. Servicio Andaluz de Salud. Manual de la gestión de casos en Andalucía: enfermeras gestoras de casos en atención primaria. Consejería de Salud. [Internet] 2007. [acessado 2019 Fev 25]. Disponível em http:// www.index-f.com/lascasas/documentos/lc0245.pdf

13. Implementación de un modelo integrado de enfermería de enlace: un estudio descriptivo. Enferm Clín 2008; 18(5):253-261.

14. España. Generalitat Valenciana. Plan para la mejora de la Atención Domiciliaria en la Comunidad Valenciana 2004-2007. Conselleria de Sanitat. [Internet] 2007 [acessado 2019 Fev 27]. Disponível em: https://www. uv.es/joguigo/materiales-dominio-profesional/PEMAD.pdf 
15. España. Generalitat Valenciana. Guía de Atención a las personas cuidadoras familiares en el ámbito sanitario. Conselleria de Sanitat. [Internet] 2014 [acessado 2019 Mar 1]. Disponível em: http://www.san.gva.es/documents/156344/0/Guia_de_atencion_a_las_personas_ cuidadoras_familiares_en_el_ambito-sanitario.pdf

16. Gobierno Basco. Estrategia para afrontar el reto de la cronicidad em Euskadi. Osakidetsa. Departamento de Sanidad y Consumo [Internet] 2010 [acessado 2019 Mar 1]. Disponível em: https://www.osakidetza.euskadi.eus/contenidos/informacion/documentos_cronicos/eu_cronic/adjuntos/EstrategiaCronicidad.pdf

17. Quintana S, Limón Ramos C, Osa García V, Campo Varela T. Cuidar en casa: la enfermera gestora de casos de pacientes paliativos hematológicos en el domicilio. Biblioteca Lascasas, [Internet] 2009 [acessado 2019 Abr 2]; 5(2):[aprox. 5 p.]. Disponível em: http://www.index -f.com/lascasas/documentos/lc0410.php

18. Gobierno Basco. Estrategia para afrontar el reto de la cronicidad em Euskadi. Anexo. Osakidetsa. Departamento de Sanidad y Consumo [Internet] 2010 [acessado 2019 Fev 28]. Disponível em: https://www.osakidetza.euskadi.eus/contenidos/informacion/osk_publicaciones/ es_publi/adjuntos/enfermeria/Anexos.pdf

19. Romero G. Organización Colegial de Enfermeras. Consejo General de Colegios Oficiales de Enfermería de España. Editorial. [Internet] 2016 [acessado $2019 \mathrm{Mar}$ 28]. Disponible en: https://www.consejogeneralenfermeria.org/sala-de-prensa/noticias/item/1939-la-gesti\%C3\%B3n-de-casos-en-enfermer\%C3\%ADa-unagran-desconocida

20. Prats-Guardiola M, López-Pisa RM, Abordaje integral de un caso complejo de diabetes mellitus en domicilio entre la gestora de casos y la enfermera comunitária. Enferm Clín 2010; 20(2):126-131.
21. Gómez MC. Intervención de la enfermera gestora de casos durante el ingreso hospitalario de pacientes con infección VIH. Rev Esp Salud Pública. 2011; 85:237-244

22. Morales JM, Gonzalo-Jiménez E, Martín-Santos FJ, Morilla-Herrera FC, Celdraán-Mañas M, Carrasco AM, García-Arrabal JJ, Toral-López I .Effectiveness of a nurse-led case management home care model in Primary Health care. A quasi experimental, controlled, multi-centre study BMC Health Services Res [Internet] 2008 [acessado 2019 Abr 2]; 23(8):193 Disponible en: http://www.biomedcentral.com/1472-6963/8/193

23. David HMSL, Acioli S, Seidl HM, Brandão OS. O enfermeiro na Atenção Básica: processo de trabalho, práticas de saúde e desafios contemporâneos. In: Mendonça MHM, Matta GC, Gondim R, Giovanella L, organizadores. Atenção Primária à Saúde no Brasil: conceitos, práticas e pesquisa. Rio de Janeiro: Editora Fiocruz; 2018. p. 337-368.

Artigo apresentado em 24/04/2019

Aprovado em 20/08/2019

Versão final apresentada em 07/10/2019 